\title{
Reef fish community structure in the islands of Paraja Bay, Pandeglang District, Banten, Indonesia
}

\author{
MUJIYANTO $^{1, \bullet}$, YAYUK SUGIANTI ${ }^{1}$, YUSUF ARIEF AFANDY ${ }^{2}$, RISNAWATI RAHAYU ${ }^{3}$, R. ARIEF \\ BUDIKUSUMA $^{3}$, ADRIANI SRI NASTITI ${ }^{1}$, AMRAN RONNY SYAM ${ }^{1}$, SRI ENDAH PURNAMANINGTYAS \\ ${ }^{1}$ Research Institute for Fisheries Resource Enhancement, Ministry of Marine Affairs and Fisheries. Jl. Cilalawi No. 1, Jatiluhur, Purwakarta 41152, West \\ Java, Indonesia. `email: antomj18@gmail.com \\ ${ }^{2}$ Directorate General of Marine Spatial Management, Ministry of Marine Affairs and Fisheries. J1. Medan Merdeka Timur No. 16, Central Jakarta 10110, \\ Jakarta, Indonesia \\ ${ }^{3}$ Local Government Marine and Fisheries Service of Banten Province. Jl. Syekh Nawawi Al-Bantani, Serang 42171, Banten, Indonesia
}

Manuscript received: 21 February 2021. Revision accepted: 25 September 2021.

\begin{abstract}
Mujiyanto, Sugianti Y, Afandy YA, Rahayu R, Bidikusuma RA, Nasriri AS, Syam AR, Purnaningtyas SE. 2021. Reef fish community structure in the islands of Paraja Bay, Pandeglang District, Banten, Indonesia. Biodiversitas 22: 4402-4413. Paraja Bay waters have five small islands that coexist with Ujung Kulon National Marine Park and act as a buffer zone for the diversity of fishery resources. This study aimed to assess the reef fishes community structure at small islands in Paraja Bay. Observations were made in 20 locations during August and September 2019 in the five small islands. Reef fishes community structure showed 106 species as included major group (54 species), indicator group ( 24 species) and target group (28 species), among five small islands, i.e. Badul, Mangir, Oar, Sumur and Umang Islands. The highest reef fishes abundance was founded in Umang Island waters. Several target fish species recorded during the study were mature fish (such as those targeted by fishermen). Only a small proportion were juveniles. Reef fish community structure was categorized as stress community to stable community with its indication was low diversity value, high evenness index, and low dominance. The low diversity index value illustrates that the level of uniformity of reef fish species in Paraja Bay waters is high. This condition is evidenced by each coral fish group's evenness index included in the main species group around 0.93 to 0.98 . The target species is 0.88 to 0.99 .
\end{abstract}

Keywords: Biodiversity, Paraja Bay, similarity analysis, species diversity

\section{INTRODUCTION}

The coral reef ecosystem is highly productive in terms of fisheries resources and other aquatic organisms. The components arranged in it are critical in this ecosystem's composition: coral reef, reef fishes, benthic community, and other microorganisms connected to their life cycle. The existence of coral reefs for some aquatic faunas like fish, molluscs, echinoderms, and seaweed are used to spawn ground, nursery ground, and feeding grounds (Johnson et al. 2019). The aquatic ecosystems of coral reefs are also quite fertile waters. Many reef fish species correlated with coral reef habitat territorial behaviour (Panggabean 2012) exhibit breeding patterns and rarely wander far from the coral reef ecosystem as a source of food and shelter from predators. The coral reef habitats, associated with aquatic and flora fauna, are important marine ecosystems with critical ecological functions and services in the waters (Rudi et al. 2009). The waters' environmental factors' is crucial to distributing fish diversity and growing reef (Saptarini et al. 2017). So that, the stability of fishes should not be separated, especially reef fishes, from the conditions of their habitat, namely coral reefs.

One of the biological resources closely related to the coral reef ecosystem is the reef fish species. Reef fish is one of the essential components of the coral reef ecosystems. In general, some species of fish reef's life will depend directly or indirectly on coral reefs' existence. But, increasing fishing activity was like overfishing, coral reef destruction, boom and tourism activities may reduce the health of live coral in a waters area. It will be a chance that an area dominated by coral reef becomes rubble, dead coral, and algae. Habitat changes in coral reef ecosystems significantly impact the number and population of reef fish within them. Changes occurring on several small islands in the Paraja Bay area may occur after the tsunami disaster in December 2018. Still, the Paraja Bay waters is in condition as "poor data", so that this research becomes a primary reference in providing information about the structure of the reef fish community. The existence of coral reefs along Badul Island, Mangir Island, Oar Island, Umang Island and Sumur Island are dominated by the type of "tabulate". This condition could be because the waters of Paraja Bay facing west will be affected by strong waves, and waves from the Sunda Strait are considered an obstacle to coral reef development.

Geographically, Paraja Bay's waters is located in the Indian Ocean area. It has a current system and water mass characteristics strongly influenced by the system developing in the Sunda Strait. Small islands in the Paraja Bay area greatly benefit the development and recruiting of fish stocks in the Sunda Strait region. The cluster of coral reefs based small islands also acts as a buffer area for the Ujung Kulon National Park's biodiversity. Along the coast 
of Paraja Bay and its small islands are coral reefs. This research aims to analyzed reef fish composition, ecological fish index, and the similarity of species known at small islands in Paraja Bay, Banten Province. This study will hope to become a primary base data to manage Paraja Bay waters coastal in the future. Besides, it would expect this study's results to be useful as input for fisheries management policy in coastal areas.

\section{MATERIALS AND METHODS}

\section{Study area}

The west coastal area of Banten Province, Indonesia has five small islands: Badul Island, Mangir Island, Oar Island, Umang Island, Sumur Island, and near Ujung Kulon National Marine Park. The small islands cluster is known administratively as the Paraja Bay region in the Pandeglang District area. Sampling was conducted from August to September 2019 in shallow waters (3-4 meters) of the small islands of Paraja Bay (Badul Island, Mangir Island, Oar Island, Sumur Island and Umang Island). Location of twenty (20) research stations at four sides of each small island. These were on the west's side, south's side, east's, and north's sides (Figure 1). The description of each sampling station on each side of the island is described in Table 1.

According to a study by English et al. (1997) and Edrus and Hadi (2020), research sampling used the visual census method. The visual census sampling methods did at a depth of 3-4 meters on each island's side (west, south, east and north). Data collected of reef fish using the belt transect method (English et al. 1997; Hill and Wilkinson 2004) along 50 meters with a line transect can be 2.5 meters right side and 2.5 meters left side (Figure 2). Placement of the transect line at a depth of 3-4 meters (shallow water) because it is closely to related the life patterns of reef fish and other organisms in coral reef ecosystems, so descriptions of the life patterns between biota can be clearly to explain (Mujiyanto et al. 2020). Identification of reef fish used the manuals provided by Kuiter and Tonozuka (2001), Tupper and Sheriff (2008) and Allen et al. (2013).

\section{Data analysis}

The arrangement among species will classify into three groups based on their function (English et al. 1997; Panggabean 2012; Brown et al. 2016; Edrus and Hadi 2020). Three groups will collect the result of reef fish data and information, and they are target species, indicator species and major species. The target fish species, which are economically important and often caught fish for consumption, the indicator fish species are types of reef fish that live on coral reef areas and become indicators of coral reefs' fertility. In contrast, the major species are fish reef species, which indicate the health of the water's environment. The main (main) is a small $5-25 \mathrm{~cm}$ large fish with various colour features known as ornamental fish.

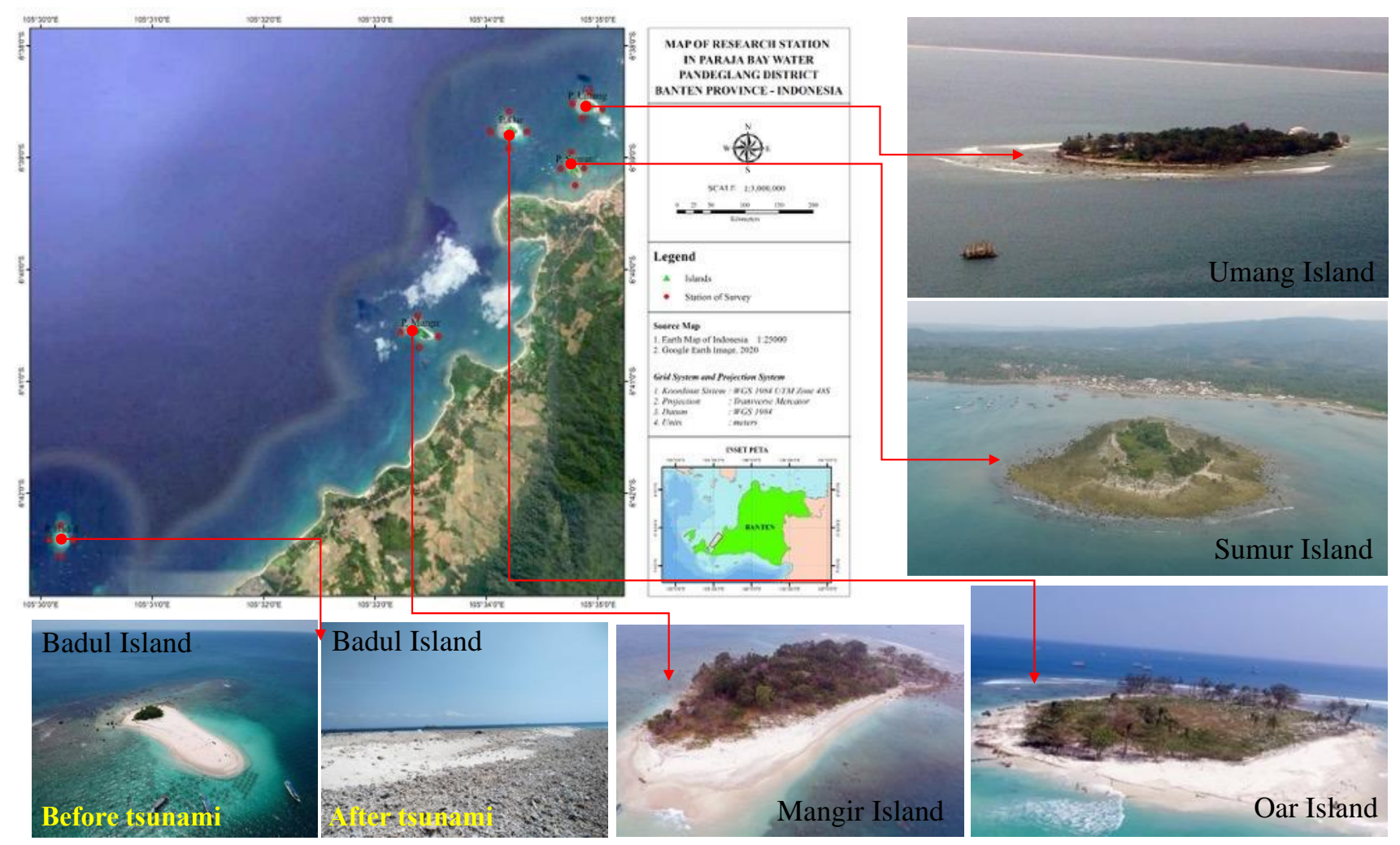

Figure 1. Map of study sites in Paraja Bay, Pandeglang District, Banten, Indonesia showing Mangir Island, Oar Island, Badul Island, Umang Island and Sumur Island) 

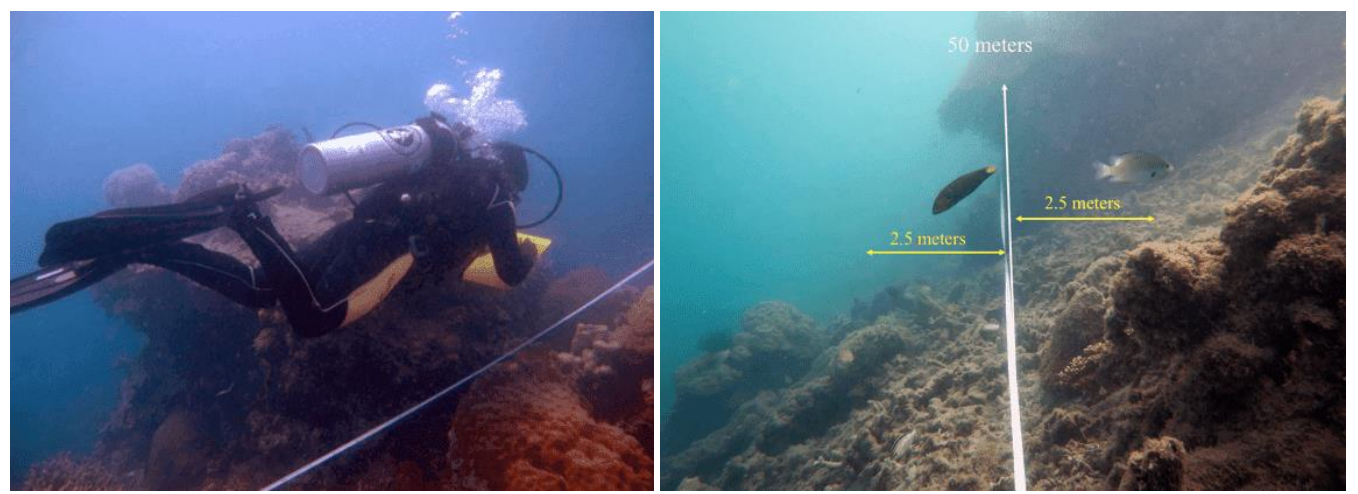

Figure 2. Observation to collect data of reef fish in Paraja Bay, Pandeglang District, Banten, Indonesia

Tabel 1. Coordination on data collection sites in the 5 small islands at Paraja Bay, Pandeglang District, Banten, Indonesia

\begin{tabular}{|c|c|c|c|c|}
\hline \multirow{2}{*}{\multicolumn{2}{|c|}{ Reserch stations }} & \multicolumn{2}{|c|}{ Geographical position } & \multirow{2}{*}{ Study period } \\
\hline & & $\mathbf{N}$ & $\mathbf{E}$ & \\
\hline \multirow[t]{4}{*}{ Mangir Island } & West & $6^{\circ} 40^{\prime} 34.99^{\prime \prime}$ & $105^{\circ} 33^{\prime} 13.18^{\prime \prime}$ & August 2019 \\
\hline & South & $6^{\circ} 40^{\prime} 42.62^{\prime \prime}$ & $105^{\circ} 33^{\prime} 26.55^{\prime \prime}$ & August 2019 \\
\hline & East & $6^{\circ} 40^{\prime} 33.83^{\prime \prime}$ & $105^{\circ} 33^{\prime} 26.51^{\prime \prime}$ & August 2019 \\
\hline & North & $6^{\circ} 40^{\prime} 28.85^{\prime \prime}$ & $105^{\circ} 33^{\prime} 18.19^{\prime}$ & August 2019 \\
\hline \multirow[t]{4}{*}{ Oar Island } & West & $6^{\circ} 38^{\prime} 47.72^{\prime \prime}$ & $105^{\circ} 34^{\prime} 04.22^{\prime \prime}$ & August 2019 \\
\hline & South & $6^{\circ} 38^{\prime} 54.51^{\prime \prime}$ & $105^{\circ} 34^{\prime} 13.08^{\prime \prime}$ & August 2019 \\
\hline & East & $6^{\circ} 38^{\prime} 47.76^{\prime \prime}$ & $105^{\circ} 34^{\prime} 18.56^{\prime \prime}$ & August 2019 \\
\hline & North & $6^{\circ} 38^{\prime} 41.60^{\prime \prime}$ & $105^{\circ} 34^{\prime} 10.10^{\prime \prime}$ & August 2019 \\
\hline \multirow[t]{4}{*}{ Badul Island } & West & $6^{\circ} 42^{\prime} 26.68^{\prime \prime}$ & $105^{\circ} 30^{\prime} 05.90^{\prime \prime}$ & September 2019 \\
\hline & South & $6^{\circ} 42^{\prime} 33.65^{\prime \prime}$ & $105^{\circ} 30^{\prime} 10.53^{\prime \prime}$ & September 2019 \\
\hline & East & $6^{\circ} 42^{\prime} 23.20^{\prime \prime}$ & $105^{\circ} 30^{\prime} 14.90^{\prime \prime}$ & September 2019 \\
\hline & North & $6^{\circ} 42^{\prime} 17.19^{\prime \prime}$ & $105^{\circ} 30^{\prime} 10.79^{\prime \prime}$ & September 2019 \\
\hline \multirow[t]{4}{*}{ Umang Island } & West & $6^{\circ} 38^{\prime} 33.15^{\prime \prime}$ & $105^{\circ} 34^{\prime} 47.20^{\prime \prime}$ & September 2019 \\
\hline & South & $6^{\circ} 38^{\prime} 38.90^{\prime \prime}$ & $105^{\circ} 34^{\prime} 51.89^{\prime \prime}$ & September 2019 \\
\hline & East & $6^{\circ} 38^{\prime} 33.11^{\prime \prime}$ & $105^{\circ} 34^{\prime} 59.19^{\prime \prime}$ & September 2019 \\
\hline & North & $6^{\circ} 38^{\prime} 33.11^{\prime \prime}$ & $105^{\circ} 34^{\prime} 52.79^{\prime \prime}$ & September 2019 \\
\hline \multirow[t]{4}{*}{ Sumur Island } & West & $6^{\circ} 39^{\prime} 07.30^{\prime \prime}$ & $105^{\circ} 34^{\prime} 40.93^{\prime \prime}$ & September 2019 \\
\hline & South & $6^{\circ} 39^{\prime} 15.83^{\prime \prime}$ & $105^{\circ} 34^{\prime} 46.84^{\prime \prime}$ & September 2019 \\
\hline & East & $6^{\circ} 39^{\prime} 07.90^{\prime \prime}$ & $105^{\circ} 34^{\prime} 50.07^{\prime \prime}$ & September 2019 \\
\hline & North & $6^{\circ} 39^{\prime} 02.93^{\prime \prime}$ & $105^{\circ} 34^{\prime} 45.06^{\prime \prime}$ & September 2019 \\
\hline
\end{tabular}

Reef fish ecological index used Shannon-Wiener (Ludwig and Reynolds 1988; Aguilar-Perera and Appeldoorn 2008), evenness index (E) Aguilar-Perera and Appeldoorn (2008), and the dominance index (D), which based on the number of species present performed by Microsoft Excel. The assessment criteria for diversity, evenness and dominance indexes of reef fish species. According to Odum and Barrett (1971), the diversity index was divided into three categories, i.e. low $\left(\mathrm{H}^{\prime}<2\right)$, moderate $\left(2 \leq \mathrm{H}^{\prime}<4\right)$ and high $(\mathrm{H} \geq 4)$ (Annas et al. 2017). Annas et al. (2017) also explain that the "C Index" is species dominance, "Pi" is the proportion of individuals found in species, " $\mathrm{i}$ " = 1 is species, and " $\mathrm{S}$ " is the number of total coral species. Further analysis to determine the level of similarity between stations using a multivariable statistical approach based on grouping species between stations and between depths, namely cluster analysis with the help of MINITAB 15 software.

\section{RESULTS AND DISCUSSION}

\section{Composition of reef fish}

Observed fish was divided into three main groups: the major species group, the target species group, the indicator species group, and the major species (English et al. 1997) (Tables 2-4). A total of 106 species of fish from 25 families were found from Badul Island, Mangir Island, Oar Island, Umang Island, and Sumur Island. Among the reef composition, with details of the 54 species were from 10 families (Table 2), 29 species were target groups from 9 families (Table 3) and indicator groups from 6 families (Tables 3-4). The top ten leading reef fish families were Gobiidae, Labridae, Pomacentridae, Apogonidae, Bleniidae, Serranidae, Murraenidae, Syngnathidae, Chaetodontidae and Lutjanidae. According to English et al. (1994) and Riskiani et al. (2019), these reef fish have been grouped according to their status into three groups: target fish, large fish and indicator fish. Costello et al. (2005) stated that as 
many as 113 fish families are coral dwellers and mostly form Perciformes. The ten main families of these reef fish were Gobiidae, Labridae, Pomacentridae, Apogonidae, Bleniidae, Serranidae, Murraenidae, Syngnathidae, Chaetodontidae and Lutjanidae (Adrim et al. 2012; Edrus and Hadi 2020). Edrus and Hadi (2020) study on the coast of Kendari in Southeast Sulawesi also showed that the composition was dominated by the large group (75\% on average), the largest group found anywhere on coral reefs. The target fish group contributed an average of $23 \%$ to the reef fish community, while the indicator fish group averaged $2 \%$.

The composition of reef fish species in the major groups with the predominance of the species found was Pomacentridae (20 species), Labridae (18 species) and Apogonidae (seven species) (Table 2). Studies by Madduppa et al. (2013) on Seribu Island found that the most common families in the remaining sub-districts were Pomacentridae (damselfishes), followed by the Labridae (wrasses). The most diverse family of reef fish found in coral reef ecosystems is Pomacentridae and Labridae. Research by Prabowo et al. (2019) about reef fish communities in three stations (Sepa Besar Island station, Papatheo Island station and Pelangi Island) in Seribu Archipelago explained the most common fish families were Pomacentridae and Labridae families. Pomacentridae family were from 23 species on Sepa Besar Island, 24 species on Papatheo Island and 20 species on Pelangi Island, and the Labridae family from 22 species on Sepa Besar Island, 12 species on Papatheo nine species on Pelangi Island.

It dominated reef fish species from the Pomacentridae family the classification analysis of the species composition of reef fish. These are Pomacentrus alexanderae and Pomacentrus molucensis. Simultaneously, those of the Labridae family were the species Hemigymnus melapterus and Coris cuvieri. The dominance of Pomacentrus alexanderae and Pomacentrus molucensis was also revealed by Prabowo et al. (2019) in Seribu Archipelago that the dominance of species from the Pomacentriade's family, these are Abudefduf sexfasciatus, Amblyglyphidodon curacao, Molucentrus alexentens and Pomacentrus alexanderea. The range of Pomacentrus alexanderae spans Australia to Japan. Type Cirrhilabrus solorensis is endogenous, even Kusuma et al. (2020) stated this is an endemic fish to Indonesia. According to (Allen and Adrim 2003), these species' distribution is only limited from Papua to Bali.

Based on the results of this study, the target fish group found were families of Acanthuridae (six species), Caesionidae (four species), Nemipteridae (five species), Serranidae (five species), Siganidae (two species), Lutjanidae (two species), Carangidae (one species), Haemulidae (one species) and Panuliridae (two species) (Table 3). The result, seen from the composition of the species for consumption fish, is the classification of the results found in line with the research results of (Edrus and Hadi 2020) on the coast of Kendari, in which found that the target group of the fish family dominates from different target groups. Fish families on the coastal area of Kendari, with the following results: families of Acanthuridae (26.7\%), Siganidae (16.7\%), Lutjanidae (13.3\%), Haemulidae, Mullidae, Scaridae, Caesionidae $6.7 \%$ each and Serranidae, Lethrinidae, Nemipteridae and Carangidae have a presence of $3.3 \%$ type, respectively. The fish species dominant found were Acanthuriada and Caesionidae families. It was in line with Paulangan et al. (2019) study, who explained that the Acanthuridae family was one of omnivorous fish that removes coral polyps to get algae that settle in the coral shelter skeleton. It was possible because of the availability of food as an indicator of presence in reef waters area. Marshell and Mumby (2015) also stated that herbivorous fish species were important in maintaining changes in coral reef area dominance by algae. The Caesionidae family was known as the yellowtail fish, found these fish on reef slopes area (about $20-30 \mathrm{~cm}$ ), and they like to be schooling (Putra et al. 2015). Also, the Caesionidae family likes coral reef areas (usually located in the column of waters). Usually, it was founded in schooling or large groups. The following fish species dominant were from Nemipteridae and Serranidae families. The Serranidae family was found commonly in and around coral reef ecosystems, which were very important to ecology because they occupy the niches of large carnivores or predators in the food chain (Ogden and Quinn 2002). Heemstra and Raddal (1993) stated that the Serranidae family occur in tropical and subtropical waters. There were around 159 species of the Serranidae family, which were from 39 species in Indonesian waters and 46 species in Southeast Asia (Astuti et al. 2016).

Different groups of target fish, such as grouper (Serranidae), snapper (Lutjanidae), Lencam (Lethrinidae), fat lips (Haemulidae), baronang (Siganidae), butane (Acanthuridae), cockatoo (Scaridae), labrid (Labridae), jackfruit seeds (Mullidae) and sand curry (Scolopsidae) are different types of reef fish common in coral reef ecosystems (Allen et al. 2013). These fish groups like coral caves, these fish groups also migrate (Allen et al. 2013; Indrawati et al. 2020). Observations on Badul Island, Mangir Island, Oar Island, Umag Island and Sumur Island show that not all types of fish groups are found in all research sites. This condition is due to the different basrelief and coral substrate from one location to another, limiting fish's spread.

This study found the presence of juvenile and adult lobsters, but they were only in the aggregate on Badul Island, Mangir Island, Oar Island, Umang Island and Sumur Island. It indicates that the coastal area of Paraja Bay is a place for lobster spawning (aggregation of spawning). The existence of widely consumed lobsters and the west coast of Banten province is one oyster trade source. However, due to regulatory issues still debated, lobster fishing efforts in the community are declining. Other fish for sale, such as Caesio cuning, Epinephelus sp., Chepalopolis sp. and Siganus sp. 
Table 2. Composition of major category of reef fish at research stations at Paraja Bay, Pandeglang District, Banten, Indonesia

\begin{tabular}{|c|c|c|c|c|c|c|c|c|c|c|c|c|c|c|c|c|c|c|c|c|c|}
\hline \multirow{2}{*}{ Familiy } & \multirow{2}{*}{ Species } & \multicolumn{4}{|c|}{ Badul Island } & \multicolumn{4}{|c|}{ Mangir Island } & \multicolumn{4}{|c|}{ Oar Island } & \multicolumn{4}{|c|}{ Umang Island } & \multicolumn{4}{|c|}{ Sumur Island } \\
\hline & & $\mathbf{W}$ & $\mathbf{S}$ & $\mathbf{E}$ & $\mathbf{N}$ & $\mathbf{W}$ & $\mathbf{S}$ & $\mathbf{E}$ & $\mathbf{N}$ & $\mathbf{W}$ & $\mathbf{S}$ & $\mathbf{E}$ & $\mathbf{N}$ & $\mathbf{W}$ & $\mathbf{S}$ & $\mathbf{E}$ & $\mathbf{N}$ & $\mathbf{W}$ & $\mathbf{S}$ & $\mathbf{E}$ & $\mathbf{N}$ \\
\hline \multirow[t]{7}{*}{ Apogonidae } & Apogon compressus & $\mathrm{xx}$ & $\mathrm{x}$ & $\mathrm{x}$ & $\mathrm{xx}$ & $\mathrm{xx}$ & & & & $\mathrm{x}$ & $\mathrm{x}$ & $\mathrm{xx}$ & $\mathrm{xx}$ & & $\mathrm{x}$ & $\mathrm{x}$ & $\mathrm{xx}$ & & & & \\
\hline & Apogon properupta & $\mathrm{x}$ & & $\mathrm{x}$ & & & & & & & $\mathrm{x}$ & & & & & $\mathrm{x}$ & & & & & \\
\hline & Archamia fucata & & & & $\mathrm{x}$ & & & $\mathrm{x}$ & $\mathrm{x}$ & $\mathrm{xx}$ & $\mathrm{xx}$ & $\mathrm{x}$ & & & $\mathrm{xx}$ & & $\mathrm{x}$ & & & & \\
\hline & Apogon frannesedai & & $\mathrm{x}$ & & & & & $\mathrm{xxx}$ & $\mathrm{x}$ & & $\mathrm{xx}$ & & $\mathrm{x}$ & & $\mathrm{x}$ & & & & & & \\
\hline & Apogon hyalosoma & $\mathrm{x}$ & $\mathrm{x}$ & & $\mathrm{xx}$ & & & & $\mathrm{x}$ & & & $\mathrm{xx}$ & & & $\mathrm{x}$ & & $\mathrm{xx}$ & $\mathrm{x}$ & & & $\mathrm{xx}$ \\
\hline & Apogon margaritophorus & & & $\mathrm{x}$ & & & & $\mathrm{xx}$ & & $\mathrm{x}$ & & & $\mathrm{x}$ & $\mathrm{x}$ & & & & & & & \\
\hline & Apogon thermalis & $\mathrm{x}$ & $\mathrm{x}$ & $\mathrm{x}$ & $\mathrm{x}$ & $\mathrm{xx}$ & & & $\mathrm{x}$ & $\mathrm{x}$ & & & & & $\mathrm{x}$ & $\mathrm{x}$ & & $\mathrm{x}$ & & & $\mathrm{x}$ \\
\hline Aulostomidae & Aulostoma chenensis & $\mathrm{x}$ & & $\mathrm{xx}$ & & $\mathrm{xx}$ & $\mathrm{x}$ & $\mathrm{x}$ & & $\mathrm{xx}$ & & & & $\mathrm{x}$ & $\mathrm{xxx}$ & & & & & & \\
\hline \multirow[t]{2}{*}{ Blennidae } & Meiacanthus anema & $\mathrm{x}$ & & & $\mathrm{xx}$ & & $\mathrm{x}$ & & $\mathrm{xx}$ & & & $\mathrm{xx}$ & $\mathrm{xx}$ & & & & $\mathrm{xx}$ & & & & \\
\hline & Salarias guttatus & & $\mathrm{x}$ & & & & & $\mathrm{xxx}$ & $\mathrm{xxx}$ & & $\mathrm{xx}$ & & $\mathrm{x}$ & & $\mathrm{x}$ & & & & & & \\
\hline Centriscidae & Aeoliscus strrigasus & & & $\mathrm{x}$ & $\mathrm{xx}$ & $\mathrm{xx}$ & & $\mathrm{xx}$ & $\mathrm{x}$ & $\mathrm{x}$ & $\mathrm{xx}$ & $\mathrm{xx}$ & $\mathrm{x}$ & & & $\mathrm{x}$ & & & & & \\
\hline Grammistidae & Diploprion bifasciatum & $\mathrm{x}$ & $\mathrm{x}$ & & $\mathrm{xx}$ & & $\mathrm{x}$ & $\mathrm{x}$ & $\mathrm{x}$ & & $\mathrm{xx}$ & & $\mathrm{x}$ & & $\mathrm{x}$ & & & & & & \\
\hline \multirow[t]{2}{*}{ Holocentridae } & Sargocentrom rubrum & $\mathrm{x}$ & & & $\mathrm{xx}$ & & & $\mathrm{x}$ & & & $\mathrm{xx}$ & & $\mathrm{xx}$ & & $\mathrm{x}$ & $\mathrm{x}$ & $\mathrm{x}$ & $\mathrm{x}$ & & & \\
\hline & Sargocentron sammara & $\mathrm{xx}$ & $\mathrm{x}$ & & & & & & $\mathrm{x}$ & & & & $\mathrm{x}$ & & $\mathrm{x}$ & & $\mathrm{x}$ & & & & \\
\hline \multirow[t]{18}{*}{ Labridae } & Bodianus mesothorax & & & & $\mathrm{xx}$ & & $\mathrm{x}$ & $\mathrm{x}$ & & & $\mathrm{xx}$ & & $\mathrm{x}$ & & $\mathrm{x}$ & & $\mathrm{x}$ & & & & \\
\hline & Cheilinus chlorourus & $\mathrm{x}$ & $\mathrm{x}$ & & $\mathrm{xx}$ & & & & $\mathrm{xx}$ & & $\mathrm{xxx}$ & & & & & & $\mathrm{x}$ & $\mathrm{x}$ & $\mathrm{x}$ & & \\
\hline & Cheilinus fasciatus & & & & $\mathrm{xx}$ & $\mathrm{x}$ & & $\mathrm{x}$ & $\mathrm{xx}$ & & $\mathrm{xx}$ & $\mathrm{x}$ & $\mathrm{x}$ & $\mathrm{x}$ & & & $\mathrm{x}$ & & $\mathrm{x}$ & & $\mathrm{x}$ \\
\hline & Cheilinus rhodochrous & $\mathrm{x}$ & & & & & $\mathrm{x}$ & & $\mathrm{x}$ & & & & & $\mathrm{x}$ & $\mathrm{x}$ & & & $\mathrm{x}$ & & & \\
\hline & Cheilinus unifasciatus & $\mathrm{x}$ & & $\mathrm{xx}$ & $\mathrm{x}$ & & $\mathrm{x}$ & & $\mathrm{x}$ & $\mathrm{xx}$ & $\mathrm{x}$ & & $\mathrm{x}$ & & $\mathrm{xx}$ & $\mathrm{xx}$ & $\mathrm{xx}$ & & & & $\mathrm{x}$ \\
\hline & Coris aygula & & & & & $\mathrm{xx}$ & & $\mathrm{x}$ & & & & $\mathrm{x}$ & & & $\mathrm{x}$ & & & & & & $\mathrm{x}$ \\
\hline & Coris cuvieri & $\mathrm{xx}$ & & $\mathrm{x}$ & $\mathrm{x}$ & $\mathrm{x}$ & & $\mathrm{xx}$ & $\mathrm{x}$ & $\mathrm{x}$ & $\mathrm{x}$ & $\mathrm{x}$ & & & $\mathrm{x}$ & $\mathrm{xx}$ & $\mathrm{xxx}$ & $\mathrm{x}$ & & & \\
\hline & Coris sp. & & & & $\mathrm{x}$ & $\mathrm{x}$ & & $\mathrm{x}$ & $\mathrm{x}$ & & $\mathrm{x}$ & $\mathrm{x}$ & $\mathrm{x}$ & & & $\mathrm{x}$ & & & & & $\mathrm{xxx}$ \\
\hline & Epibulus inssidiator & $\mathrm{xxx}$ & & & $\mathrm{x}$ & & & $\mathrm{x}$ & & & $\mathrm{x}$ & & $\mathrm{xx}$ & $\mathrm{xx}$ & & & $\mathrm{x}$ & $\mathrm{x}$ & & & \\
\hline & Ghomposus varius & & $\mathrm{x}$ & $\mathrm{x}$ & & & $\mathrm{xx}$ & & $\mathrm{x}$ & $\mathrm{x}$ & & & $\mathrm{x}$ & & & $\mathrm{x}$ & & & & & \\
\hline & Halichoeres dussumieri & & & $\mathrm{x}$ & & $\mathrm{x}$ & & & & $\mathrm{x}$ & & $\mathrm{x}$ & $\mathrm{x}$ & $\mathrm{xx}$ & $\mathrm{x}$ & $\mathrm{xx}$ & & & $\mathrm{x}$ & & \\
\hline & Halichoeres javanicus & $\mathrm{x}$ & & & $\mathrm{x}$ & $\mathrm{x}$ & $\mathrm{x}$ & $\mathrm{xx}$ & & & $\mathrm{x}$ & $\mathrm{x}$ & & & & & $\mathrm{x}$ & & $\mathrm{x}$ & & $\mathrm{xx}$ \\
\hline & Halichoeres melamurus & & & & $\mathrm{x}$ & $\mathrm{x}$ & & $\mathrm{x}$ & & & $\mathrm{x}$ & $\mathrm{x}$ & & $\mathrm{x}$ & & & $\mathrm{x}$ & & & & $\mathrm{xx}$ \\
\hline & Hemigymnus melapterus & & $\mathrm{xx}$ & $\mathrm{xx}$ & $\mathrm{xx}$ & $\mathrm{xxx}$ & $\mathrm{xx}$ & $\mathrm{x}$ & $\mathrm{xx}$ & $\mathrm{xx}$ & $\mathrm{xx}$ & $\mathrm{xxx}$ & $\mathrm{x}$ & & & & & & & & \\
\hline & Hemygymnus fasciatus & $\mathrm{x}$ & $\mathrm{x}$ & & & & & & $\mathrm{x}$ & & & & & & & & & & & & $\mathrm{x}$ \\
\hline & Labrichthys unitineatus & $\mathrm{x}$ & $\mathrm{x}$ & $\mathrm{xx}$ & $\mathrm{xxx}$ & & & $\mathrm{xxx}$ & $\mathrm{x}$ & $\mathrm{xx}$ & $\mathrm{xxx}$ & & $\mathrm{x}$ & & & & & & $\mathrm{x}$ & & \\
\hline & Labroides bicolor & & & $\mathrm{x}$ & & & $\mathrm{xx}$ & $\mathrm{xxx}$ & & $\mathrm{x}$ & & & & & & & & & & & \\
\hline & Labroides dimidiatus & & & & $\mathrm{x}$ & $\mathrm{x}$ & $\mathrm{x}$ & $\mathrm{xxx}$ & & & $\mathrm{x}$ & $\mathrm{x}$ & $\mathrm{xxx}$ & & & & & & & & \\
\hline Pinguipedidae & Parapercis hexaphtalma & $\mathrm{xx}$ & & $\mathrm{x}$ & $\mathrm{x}$ & $\mathrm{x}$ & & & $\mathrm{xx}$ & & $\mathrm{x}$ & & & & & & $\mathrm{x}$ & $\mathrm{x}$ & & & \\
\hline \multirow[t]{12}{*}{ Pomacentridae } & Abudaefdus vaigiensis & $\mathrm{xxx}$ & & $\mathrm{xxx}$ & & & & $\mathrm{x}$ & & & $\mathrm{x}$ & & & $\mathrm{x}$ & & $\mathrm{xx}$ & $\mathrm{xx}$ & & & & $\mathrm{x}$ \\
\hline & Amblygkyphidodon curacao & $\mathrm{x}$ & & $\mathrm{x}$ & & $\mathrm{xx}$ & $\mathrm{xx}$ & & $\mathrm{x}$ & & & & $\mathrm{x}$ & & $\mathrm{x}$ & $\mathrm{xx}$ & $\mathrm{xx}$ & & & & \\
\hline & Amblyglyphidodon leucogaster & $\mathrm{x}$ & & $\mathrm{x}$ & & $\mathrm{x}$ & $\mathrm{x}$ & $\mathrm{x}$ & & $\mathrm{xx}$ & & $\mathrm{x}$ & & $\mathrm{x}$ & & & $\mathrm{x}$ & & & & \\
\hline & Amphiprion clarkiii & & & & $\mathrm{xx}$ & & & $\mathrm{x}$ & $\mathrm{x}$ & & $\mathrm{x}$ & & & $\mathrm{x}$ & $\mathrm{x}$ & & $\mathrm{x}$ & & & & \\
\hline & Amphiprion ocelaris & & & $\mathrm{x}$ & & & & & & $\mathrm{x}$ & & $\mathrm{x}$ & & & & & $\mathrm{xx}$ & & & & $\mathrm{x}$ \\
\hline & Amphiprion sandracinos & $\mathrm{x}$ & & & $\mathrm{xx}$ & & $\mathrm{xx}$ & $\mathrm{xx}$ & $\mathrm{xxx}$ & $\mathrm{xx}$ & $\mathrm{x}$ & $\mathrm{xx}$ & $\mathrm{xx}$ & $\mathrm{xx}$ & $\mathrm{xxx}$ & $\mathrm{x}$ & & & & & \\
\hline & Chromis delta & $\mathrm{x}$ & & $\mathrm{x}$ & $\mathrm{x}$ & $\mathrm{xx}$ & & & & & & $\mathrm{x}$ & & & & & & & & & \\
\hline & Chromis leucura & $\mathrm{x}$ & & $\mathrm{x}$ & & $\mathrm{x}$ & $\mathrm{xx}$ & $\mathrm{xxx}$ & & & $\mathrm{xx}$ & $\mathrm{x}$ & $\mathrm{xx}$ & $\mathrm{xxx}$ & & & & & & & \\
\hline & Chromis viridis & & & & $\mathrm{x}$ & & $\mathrm{xx}$ & & & & $\mathrm{xx}$ & $\mathrm{xx}$ & & $\mathrm{x}$ & & $\mathrm{x}$ & & & & & \\
\hline & Chrysiptera & $\mathrm{xx}$ & & $\mathrm{xxx}$ & $\mathrm{xx}$ & $\mathrm{x}$ & & $\mathrm{xx}$ & $\mathrm{xx}$ & $\mathrm{xxx}$ & $\mathrm{x}$ & & $\mathrm{xxx}$ & & & & & & & & \\
\hline & Chrysiptera talboti & & & & & & $\mathrm{xxx}$ & & & & & & $\mathrm{xx}$ & & & & & & & & \\
\hline & $\begin{array}{l}\text { Dascyltus trimaculatus } \\
\text { Dischistodus prosopotaenia }\end{array}$ & $\mathrm{xxx}$ & & & & $\mathrm{xxx}$ & $\mathrm{x}$ & $\mathrm{xx}$ & $\mathrm{xxx}$ & & $\mathrm{x}$ & $\begin{array}{c}\mathrm{xx} \\
\mathrm{x}\end{array}$ & $\mathrm{xxx}$ & & & & $\mathrm{x}$ & & & & \\
\hline
\end{tabular}




\begin{tabular}{|c|c|c|c|c|c|c|c|c|c|c|c|c|c|c|c|c|c|c|c|c|}
\hline & Neoglyphidodon thotacotaeniatus & & & $\mathrm{x}$ & & $\mathrm{x}$ & & $\mathrm{x}$ & $\mathrm{x}$ & $\mathrm{x}$ & & $\mathrm{xx}$ & $\mathrm{x}$ & $\mathrm{x}$ & $\mathrm{x}$ & $\mathrm{x}$ & & & & $\mathrm{xxx}$ \\
\hline & Neopomacentrus azysron & $\mathrm{x}$ & & $\mathrm{x}$ & & $\mathrm{xx}$ & $\mathrm{x}$ & & $\mathrm{x}$ & $\mathrm{x}$ & & $\mathrm{x}$ & $\mathrm{x}$ & & $\mathrm{x}$ & $\mathrm{x}$ & $\mathrm{x}$ & & & \\
\hline & Plectroglyphidodon lacrymatus & & & $\mathrm{x}$ & & $\mathrm{x}$ & $\mathrm{xx}$ & & $\mathrm{x}$ & & & $\mathrm{x}$ & & & $\mathrm{x}$ & & $\mathrm{xx}$ & & & $\mathrm{x}$ \\
\hline & Pomacentrus alexanderae & & $\mathrm{x}$ & & & $\mathrm{x}$ & & $\mathrm{x}$ & & & $\mathrm{xx}$ & & $\mathrm{x}$ & $\mathrm{x}$ & & & $\mathrm{x}$ & & & \\
\hline & Pomacentrus bahkanensis & $\mathrm{x}$ & & $\mathrm{x}$ & & & & & $\mathrm{x}$ & & $\mathrm{xx}$ & & $\mathrm{x}$ & & $\mathrm{x}$ & & & $\mathrm{x}$ & $\mathrm{x}$ & \\
\hline & Pomacentrus moluccensis & $\mathrm{x}$ & & $\mathrm{x}$ & $\mathrm{x}$ & $\mathrm{xx}$ & & & $\mathrm{x}$ & $\mathrm{x}$ & & $\mathrm{x}$ & & & $\mathrm{x}$ & $\mathrm{x}$ & & & & \\
\hline Tetraodontidae & $\begin{array}{l}\text { Pomacentrus philipinus } \\
\text { Canthigaster valentini }\end{array}$ & $\mathrm{x}$ & & $\begin{array}{l}\mathrm{x} \\
\mathrm{x}\end{array}$ & $\mathrm{X}$ & $\mathrm{x}$ & $\mathrm{x}$ & & & & $\mathrm{x}$ & $\mathrm{x}$ & $\mathrm{x}$ & & & & $\mathrm{x}$ & & $\mathrm{x}$ & $\mathrm{xx}$ \\
\hline
\end{tabular}

Note: $x$ : 1-3 individuals; xx: 4-6 individuals; xxx: > 7 individuals.

Table 3. Composition of target category of reef fish at research stations at Paraja Bay, Pandeglang District, Banten, Indonesia

\begin{tabular}{|c|c|c|c|c|c|c|c|c|c|c|c|c|c|c|c|c|c|c|c|c|c|}
\hline \multirow{2}{*}{ Familiy } & \multirow{2}{*}{ Species } & \multicolumn{4}{|c|}{ Badul Island } & \multicolumn{4}{|c|}{ Mangir Island } & \multicolumn{4}{|c|}{ Oar Island } & \multicolumn{4}{|c|}{ Umang Island } & \multicolumn{4}{|c|}{ Sumur Island } \\
\hline & & $\mathbf{W}$ & $\mathbf{S}$ & $\mathbf{E}$ & $\mathbf{N}$ & W & $\mathbf{S}$ & $\mathbf{E}$ & $\mathbf{N}$ & W & $\mathbf{S}$ & $\mathbf{E}$ & $\mathbf{N}$ & $\mathbf{W}$ & $\mathbf{S}$ & $\mathbf{E}$ & $\mathbf{N}$ & $\mathbf{W}$ & $\mathbf{S}$ & $\mathbf{E}$ & $\mathbf{N}$ \\
\hline \multirow[t]{6}{*}{ Acanthuridae } & Acanthurus blochii & & & $\mathrm{x}$ & & $\mathrm{x}$ & & & & $\mathrm{xx}$ & & & $\mathrm{x}$ & & & $\mathrm{x}$ & & & & & $\mathrm{xx}$ \\
\hline & Acanthurus sp. & $\mathrm{x}$ & & $\mathrm{x}$ & & $\mathrm{xx}$ & $\mathrm{xx}$ & $\mathrm{x}$ & $\mathrm{x}$ & $\mathrm{x}$ & & & $\mathrm{x}$ & $\mathrm{xx}$ & $\mathrm{xxx}$ & $\mathrm{xx}$ & $\mathrm{xx}$ & $\mathrm{xx}$ & $\mathrm{xxx}$ & & \\
\hline & Acanthurus tristis & $\mathrm{x}$ & $\mathrm{x}$ & $\mathrm{x}$ & & $\mathrm{x}$ & $\mathrm{x}$ & & $\mathrm{x}$ & $\mathrm{x}$ & $\mathrm{x}$ & & & $\mathrm{x}$ & & $\mathrm{x}$ & & & & & \\
\hline & Ctenochaetus binotatus & & & & & $\mathrm{x}$ & & & $\mathrm{x}$ & & $\mathrm{xx}$ & $\mathrm{xx}$ & & & $\mathrm{x}$ & $\mathrm{x}$ & $\mathrm{xx}$ & $\mathrm{xxx}$ & & & \\
\hline & Ctenochaetus strigasus & $\mathrm{x}$ & & & $\mathrm{x}$ & & $\mathrm{x}$ & $\mathrm{x}$ & & & $\mathrm{x}$ & & & $\mathrm{x}$ & & $\mathrm{x}$ & $\mathrm{xx}$ & & & & \\
\hline & Zebrasoma scopas & $\mathrm{x}$ & & $\mathrm{x}$ & & & & $\mathrm{x}$ & & $\mathrm{x}$ & $\mathrm{x}$ & $\mathrm{xx}$ & $\mathrm{xxx}$ & $\mathrm{xx}$ & & & $\mathrm{x}$ & & & & \\
\hline \multirow[t]{4}{*}{ Caesionidae } & Caesio caerulaureus & $\mathrm{x}$ & $\mathrm{x}$ & $\mathrm{x}$ & & $\mathrm{x}$ & $\mathrm{xxx}$ & & $\mathrm{x}$ & $\mathrm{x}$ & & $\mathrm{x}$ & $\mathrm{xxx}$ & & & & $\mathrm{xx}$ & & & & \\
\hline & Caesio teres & & & $\mathrm{x}$ & & $\mathrm{x}$ & & & & $\mathrm{xxx}$ & & & $\mathrm{x}$ & & & & & $\mathrm{x}$ & & & \\
\hline & Pterocaesio pisang & $\mathrm{x}$ & $\mathrm{x}$ & $\mathrm{x}$ & & $\mathrm{xx}$ & $\mathrm{xx}$ & $\mathrm{x}$ & $\mathrm{x}$ & $\mathrm{x}$ & & & $\mathrm{x}$ & $\mathrm{x}$ & $\mathrm{xxx}$ & $\mathrm{x}$ & & & & & \\
\hline & Pterocaesio tile & & $\mathrm{x}$ & $\mathrm{x}$ & & $\mathrm{x}$ & $\mathrm{x}$ & & $\mathrm{x}$ & $\mathrm{x}$ & $\mathrm{x}$ & & & $\mathrm{x}$ & & $\mathrm{xx}$ & $\mathrm{xx}$ & $\mathrm{x}$ & & & \\
\hline Carangidae & Caranx bajad & $\mathrm{x}$ & & $\mathrm{x}$ & & & & $\mathrm{x}$ & & $\mathrm{x}$ & $\mathrm{x}$ & $\mathrm{xx}$ & $\mathrm{xx}$ & $\mathrm{xx}$ & & & $\mathrm{x}$ & & & & $\mathrm{x}$ \\
\hline Haemulidae & Plectorhynchus chaetodontoides & & $\mathrm{x}$ & $\mathrm{x}$ & & $\mathrm{x}$ & $\mathrm{x}$ & & $\mathrm{x}$ & $\mathrm{x}$ & & $\mathrm{x}$ & $\mathrm{x}$ & & & & $\mathrm{x}$ & $\mathrm{x}$ & & & $\mathrm{x}$ \\
\hline \multirow[t]{2}{*}{ Lutjanidae } & Lutjanus biggutatus & & & $\mathrm{x}$ & & $\mathrm{x}$ & & & & $\mathrm{x}$ & & & $\mathrm{x}$ & & & $\mathrm{x}$ & & & & & \\
\hline & Lutjanus vitta & $\mathrm{x}$ & $\mathrm{x}$ & $\mathrm{x}$ & & $\mathrm{xx}$ & $\mathrm{x}$ & $\mathrm{x}$ & $\mathrm{x}$ & $\mathrm{x}$ & & & $\mathrm{x}$ & $\mathrm{xx}$ & $\mathrm{xxx}$ & $\mathrm{x}$ & $\mathrm{xx}$ & & & & \\
\hline \multirow[t]{5}{*}{ Nemipteridae } & Scolopsis bilineatus & $\mathrm{x}$ & & & $\mathrm{xx}$ & & $\mathrm{x}$ & $\mathrm{x}$ & & & $\mathrm{x}$ & & & $\mathrm{x}$ & & $\mathrm{x}$ & $\mathrm{xx}$ & $\mathrm{xxx}$ & & & \\
\hline & Scolopsis ciliatus & $\mathrm{x}$ & & $\mathrm{x}$ & & & & $\mathrm{x}$ & & $\mathrm{x}$ & $\mathrm{x}$ & $\mathrm{xx}$ & $\mathrm{xx}$ & $\mathrm{xx}$ & & & $\mathrm{x}$ & & & & \\
\hline & Scolopsis margaritifer & $\mathrm{x}$ & & & $\mathrm{xx}$ & & $\mathrm{x}$ & & $\mathrm{x}$ & & & $\mathrm{x}$ & $\mathrm{x}$ & & & & & $\mathrm{x}$ & & & \\
\hline & Scolopsis trilineatus & $\mathrm{x}$ & & $\mathrm{x}$ & $\mathrm{x}$ & $\mathrm{xx}$ & & & $\mathrm{x}$ & $\mathrm{x}$ & & & & & $\mathrm{x}$ & & $\mathrm{x}$ & & & & \\
\hline & Scolopsis vosmeri & $\mathrm{x}$ & & $\mathrm{x}$ & & $\mathrm{x}$ & & & $\mathrm{x}$ & $\mathrm{x}$ & & $\mathrm{x}$ & $\mathrm{x}$ & & & & $\mathrm{x}$ & & & & \\
\hline \multirow[t]{5}{*}{ Serranidae } & Cephalopholis boenack & & & & & & $\mathrm{x}$ & & & & & & $\mathrm{xxx}$ & & & & & & & & \\
\hline & Cephalopholis cyanostigma & $\mathrm{xx}$ & & & & $\mathrm{xx}$ & $\mathrm{x}$ & $\mathrm{xx}$ & $\mathrm{xxx}$ & & $\mathrm{xx}$ & $\mathrm{xx}$ & $\mathrm{xxx}$ & & & & $\mathrm{x}$ & & & & \\
\hline & Cephalopholis microprion & $\mathrm{x}$ & & & $\mathrm{x}$ & & & & & $\mathrm{x}$ & & & & $\mathrm{x}$ & & & & $\mathrm{x}$ & & & \\
\hline & Cephalopholis miniata & $\mathrm{x}$ & & & $\mathrm{x}$ & & & & & & & & & & & & & & & & \\
\hline & Epinephelus fasciatus & & & & & $\mathrm{x}$ & & & & & & & & $\mathrm{x}$ & & & & $\mathrm{x}$ & & & \\
\hline \multirow[t]{2}{*}{ Siganidae } & Siganus javus & & & $\mathrm{x}$ & & $\mathrm{x}$ & & & & $\mathrm{x}$ & & & $\mathrm{x}$ & & & & & $\mathrm{x}$ & & & \\
\hline & Siganus virgatus & $\mathrm{x}$ & $\mathrm{x}$ & $\mathrm{x}$ & & $\mathrm{xx}$ & $\mathrm{x}$ & $\mathrm{x}$ & $\mathrm{x}$ & $\mathrm{x}$ & & & $\mathrm{x}$ & $\mathrm{xx}$ & $\mathrm{xxx}$ & $\mathrm{x}$ & & & & & \\
\hline \multirow[t]{2}{*}{ Palinuridae } & Panulirus sp. (juvenile) & & & & $\mathrm{x}$ & & $\mathrm{x}$ & & & & $\mathrm{x}$ & & $\mathrm{x}$ & $\mathrm{x}$ & & & & $\mathrm{x}$ & & & \\
\hline & Panulirus versicolor (adult) & & & $\mathrm{x}$ & $\mathrm{x}$ & & & & $\mathrm{x}$ & & & $\mathrm{x}$ & & $\mathrm{x}$ & & & & $\mathrm{x}$ & & & \\
\hline
\end{tabular}

Note: $x: 1-3$ individuals; $x x: 4-6$ individuals; $x x x:>7$ individuals. 
Table 4. Composition of indicator category of reef fish at research stations at Paraja Bay, Pandeglang District, Banten, Indonesia

\begin{tabular}{|c|c|c|c|c|c|c|c|c|c|c|c|c|c|c|c|c|c|c|c|c|c|}
\hline \multirow{2}{*}{ Familiy } & \multirow{2}{*}{ Species } & \multicolumn{4}{|c|}{ Badul Island } & \multicolumn{4}{|c|}{ Mangir Island } & \multicolumn{4}{|c|}{ Oar Island } & \multicolumn{4}{|c|}{ Umang Island } & \multicolumn{4}{|c|}{ Sumur Island } \\
\hline & & $\mathbf{W}$ & $\mathbf{S}$ & $\mathbf{E}$ & $\mathbf{N}$ & $\mathbf{W}$ & $\mathbf{S}$ & $\mathbf{E}$ & $\mathbf{N}$ & $\mathbf{W}$ & $\mathbf{S}$ & $\mathbf{E}$ & $\mathbf{N}$ & $\mathbf{W}$ & $\mathbf{S}$ & $\mathbf{E}$ & $\mathbf{N}$ & $\mathbf{W}$ & $\mathbf{S}$ & $\mathbf{E}$ & $\mathbf{N}$ \\
\hline \multirow[t]{2}{*}{ Balistidae } & Balistapus undulatus & $\mathrm{x}$ & $\mathrm{x}$ & & $\mathrm{xx}$ & & $\mathrm{x}$ & & $\mathrm{x}$ & & & $\mathrm{x}$ & $\mathrm{x}$ & & & $\mathrm{x}$ & & $\mathrm{x}$ & & & $\mathrm{x}$ \\
\hline & Sufflamen fraenatus & $\mathrm{x}$ & $\mathrm{x}$ & $\mathrm{x}$ & $\mathrm{x}$ & $\mathrm{xx}$ & & & $\mathrm{x}$ & $\mathrm{x}$ & & & & & $\mathrm{x}$ & & & $\mathrm{x}$ & & & \\
\hline \multirow[t]{11}{*}{ Chaetodontidae } & Chaetodon citrinellus & $\mathrm{xx}$ & $\mathrm{xx}$ & $\mathrm{xxx}$ & $\mathrm{xx}$ & $\mathrm{xx}$ & $\mathrm{xx}$ & $\mathrm{xx}$ & $\mathrm{xx}$ & $\mathrm{xxx}$ & $\mathrm{x}$ & & $\mathrm{xxx}$ & & & $\mathrm{xx}$ & $\mathrm{xx}$ & & & & \\
\hline & Chaetodon collare & & & & & & $\mathrm{x}$ & & & & & & $\mathrm{xxx}$ & & & $\mathrm{x}$ & & & & & \\
\hline & Chaetodon kleinii & $\mathrm{xx}$ & $\mathrm{xxx}$ & & & $\mathrm{xx}$ & $\mathrm{x}$ & $\mathrm{xx}$ & $\mathrm{xxx}$ & & $\mathrm{xxx}$ & $\mathrm{xx}$ & $\mathrm{xxx}$ & & & $\mathrm{x}$ & $\mathrm{xx}$ & $\mathrm{xxx}$ & & & \\
\hline & Chaetodon lunula & $\mathrm{x}$ & & & $\mathrm{x}$ & & & & & & & & & & & & & & & & \\
\hline & Chaetodon melanotus & & & $\mathrm{x}$ & & $\mathrm{x}$ & $\mathrm{x}$ & $\mathrm{xx}$ & $\mathrm{xx}$ & $\mathrm{xx}$ & & & & & & & & & & & \\
\hline & Chaetodon rafflesii & & & & $\mathrm{x}$ & $\mathrm{x}$ & $\mathrm{xxx}$ & & & & & & & $\mathrm{x}$ & & & $\mathrm{x}$ & & & & \\
\hline & Chaetodon trifascialis & & & & $\mathrm{x}$ & $\mathrm{x}$ & & $\mathrm{x}$ & $\mathrm{x}$ & $\mathrm{x}$ & & & & & & $\mathrm{x}$ & & & & & \\
\hline & Chaetodon sp. & & & & $\mathrm{x}$ & & $\mathrm{xx}$ & $\mathrm{xx}$ & & $\mathrm{x}$ & & & & $\mathrm{x}$ & & & & & & & \\
\hline & Heniochus pleurotaenia & & & & & & & $\mathrm{x}$ & & $\mathrm{x}$ & $\mathrm{x}$ & $\mathrm{xx}$ & $\mathrm{xx}$ & $\mathrm{xx}$ & & $\mathrm{x}$ & & & & & $\mathrm{x}$ \\
\hline & Heniochus singularius & & & $\mathrm{x}$ & $\mathrm{xxx}$ & & $\mathrm{x}$ & & $\mathrm{x}$ & & & $\mathrm{x}$ & $\mathrm{x}$ & & & $\mathrm{xx}$ & $\mathrm{xx}$ & & & & \\
\hline & Heniochus varius & & & & & & & & $\mathrm{x}$ & $\mathrm{x}$ & & & & & & $\mathrm{x}$ & & & & & \\
\hline \multirow[t]{2}{*}{ Pomacanthidae } & Centropyge eibli & $\mathrm{x}$ & $\mathrm{x}$ & $\mathrm{x}$ & $\mathrm{x}$ & $\mathrm{xx}$ & $\mathrm{xx}$ & $\mathrm{xx}$ & $\mathrm{xx}$ & $\mathrm{xxx}$ & $\mathrm{x}$ & & $\mathrm{xxx}$ & & $\mathrm{xxx}$ & $\mathrm{x}$ & & & & & \\
\hline & Pomacanthus semicirculatus & & $\mathrm{x}$ & $\mathrm{x}$ & & $\mathrm{x}$ & $\mathrm{x}$ & & & & & & $\mathrm{xxx}$ & & & $\mathrm{xx}$ & $\mathrm{xx}$ & $\mathrm{xx}$ & & & \\
\hline \multirow[t]{7}{*}{ Scaridae } & Cetoscarus bicolor & $\mathrm{x}$ & & $\mathrm{x}$ & & & $\mathrm{xxx}$ & $\mathrm{x}$ & & $\mathrm{x}$ & $\mathrm{x}$ & $\mathrm{xx}$ & $\mathrm{x}$ & $\mathrm{xx}$ & & & $\mathrm{x}$ & & & & \\
\hline & Chlorurus sordidus & $\mathrm{x}$ & & & $\mathrm{xx}$ & & $\mathrm{x}$ & & $\mathrm{x}$ & & & $\mathrm{x}$ & $\mathrm{x}$ & & & & $\mathrm{x}$ & & & & $\mathrm{xx}$ \\
\hline & Scarus bleekeri & $\mathrm{x}$ & & $\mathrm{x}$ & $\mathrm{x}$ & $\mathrm{xx}$ & & & $\mathrm{x}$ & $\mathrm{x}$ & & & & & $\mathrm{x}$ & $\mathrm{x}$ & & $\mathrm{x}$ & & & $\mathrm{x}$ \\
\hline & Scarus dimidiatus & $\mathrm{x}$ & & $\mathrm{x}$ & $\mathrm{xx}$ & $\mathrm{x}$ & $\mathrm{x}$ & & $\mathrm{x}$ & $\mathrm{x}$ & & $\mathrm{x}$ & $\mathrm{xx}$ & & & & & & & & \\
\hline & Scarus ghobban & & & $\mathrm{x}$ & $\mathrm{x}$ & $\mathrm{xx}$ & $\mathrm{xxx}$ & & & $\mathrm{xxx}$ & & & $\mathrm{x}$ & & & & & & & & $\mathrm{x}$ \\
\hline & Scarus niger & $\mathrm{x}$ & $\mathrm{x}$ & $\mathrm{x}$ & $\mathrm{xx}$ & $\mathrm{xx}$ & $\mathrm{xx}$ & $\mathrm{x}$ & $\mathrm{x}$ & $\mathrm{x}$ & & & $\mathrm{x}$ & $\mathrm{xx}$ & $\mathrm{xxx}$ & $\mathrm{x}$ & & & & & \\
\hline & Scarus rubroviolaceus & & $\mathrm{x}$ & $\mathrm{x}$ & & $\mathrm{x}$ & $\mathrm{x}$ & & $\mathrm{x}$ & $\mathrm{x}$ & $\mathrm{x}$ & & & $\mathrm{x}$ & & $\mathrm{xx}$ & $\mathrm{xx}$ & $\mathrm{xx}$ & & & \\
\hline Scorpaenidae & Pterois volitans & $\mathrm{x}$ & & $\mathrm{x}$ & $\mathrm{x}$ & & & $\mathrm{x}$ & & $\mathrm{x}$ & $\mathrm{x}$ & $\mathrm{xx}$ & & $\mathrm{xx}$ & & & $\mathrm{x}$ & & & & \\
\hline Zanclidae & Zanclus cornotus & & $\mathrm{x}$ & $\mathrm{x}$ & & $\mathrm{x}$ & & & $\mathrm{x}$ & $\mathrm{x}$ & & $\mathrm{x}$ & $\mathrm{x}$ & & & & $\mathrm{xx}$ & & & & \\
\hline
\end{tabular}

Note: x: 1-3 individuals; xx: 4-6 individuals; xxx: > 7 individuals. 
The result of saving the composition of reef fish included in the indicator fish group showed that the study site's dominant fish were the Chaetodontidae family of 10 species. In addition to the Chatodontidae family, several reef fish have also been included in the indicator species category of the Pomacanthidae family. Species from these two families were found to dominate the presence of field observations, namely Chaetodon citrinellus (16 individuals) and Centropyge eibli (52 individuals). Several studies have shown that this species has a high tolerance for adaptation to different coral reef habitat conditions due to its omnivorous diet (Bejarano et al. 2019). Also found in Wibowo et al. (2013) has research results, stating that the reef fish community in the Seribu Islands found 19 species of the Chatodontidae family. Adrim (2007) and Wibowo et al. (2013) found the composition of coral fish species around the Sunda Strait waters as many as 32 species, on the Enggano Islands, in Bengkulu province as many as 30 species (Adrim 2007). Besides, Wibowo et al. (2013) added that the diversity of Chaetodontidae in Banda was determined by the excellent diversity index of coral reefs in the region. As many as 194 coral species have been found in Banggai waters (Siringoringo and Hadi 2015; Indrawati et al. 2020), and more than 300 coral species have been counted from Lamalera (Bachtiar et al. 2012).

Bellwood et al. (2010) state that many reef fish of the Chaetodontidae family eat coral polyps so that if the coral reefs in an area are healthy, they will invite these fish to live in the area because they have sufficient food. Besides, roughness significantly influences species diversity (Gratwicke and Speight 2005). Thus, the presence of eight coralivore fish indicates that the presence of live corals related to coral reefs' health in the Paraja Bay region's small islands was a poor condition. It needs specific research to know specific reef health conditions in Paraja Bay waters.

\section{Ecological index of reef fish}

Based on the biodiversity index $\left(\mathrm{H}^{\prime}\right)$, found to be generally to category as high diversity, namely $\mathrm{H}^{\prime}<2$, which ranges from 0.83 to 1.44 (major group), 0.27 to 1.21 (target group) and 0.53 to 1.14 (indicator group) (Table 4). The low diversity index value illustrates that the level of uniformity of reef fish species in Paraja Bay waters is high. This condition is evidenced by each coral fish group's evenness index included in the main species group around 0.93 to 0.98 . The target species is 0.88 to 0.99 . The species indicator has a value range of 0.01 to 0.07 . The value of evenness index found in 18 research stations indicated that complex coral communities were in a stable state, whereas South Sumur Island is in community stressed condition. Meanwhile, during research observation, the team did not succeed in sampling in East Sumur Island. Because the east side of the island well is in shallow condition due to activities from the mainland, based on Krebs (1972) criteria and Yuanike et al. (2019) $0<\mathrm{E} \leq$ 0.50: stressed community, $0.50<\mathrm{E} \leq 0.75$ : unstable community and 0.75 $<\mathrm{E} \leq 1.50$ : stable community.

Based on the value of the diversity index produced of reef fish community from various selected stations on each side of Badul Island, Mangir Island, Oar Island, Umang Island and Sumur Island, the community is a stressed community to be stable. The highest value of evenness index (0.99) was obtained on the south side of Badul Island, indicating that the fish were the most diverse, evenly distributed distribution of fish species. There was no individual concentration of one species, indicating that the ecological water conditions at that location were relatively stable compared to the other areas.

The species dominance values were found showed values ranging from 0.01 to 0.08 (major group), 0.01 to 0.10 (target group) and 0.01 to 0.07 (indicator group) (Table 4). According to Odum and Barrett (1971), the range of dominance index values is 0 to 1 . If the dominance index value is close to 1 (one), one species dominates in the community in one community. On the other hand, if the dominance index value is close to "zero", then there is no single species dominating the community in one community. Mardasin et al. (2011) also explained that the value of the dominance index range, namely if the value of $\mathrm{Di}=0$ means that the level of similarity is low and if $\mathrm{Di}=$ 1 means that of agreement (Ludwig and Reynolds 1988). Arifin et al. (2018) and Yuanike et al. (2019) explained that Dominance Index (D) describes the presence or absence of dominant species. The calculation results showed that the dominance value was closer to 0 (zero) than 1 (one), which was in the range of 0.03 to 0.09 . Therefore, it can be said that, in general, there were no species that dominated the coral reef ecosystem in the Paraja Bay area and were categorized as low.

\section{Similirarity index of species reef fish species}

The results of the cluster analysis showed that the similarity index was grouped into three groups of entities (based on the function of the group), namely the major species group (Figure 3), target species group (Figure 4) and indicator fish group (Figure 5). The dendrogram cluster analysis results on large fish groups showed the stations with the most significant similarity $(79.07 \%)$ at the East Badul Island and West Oar Island stations. Also found similarities of reef fish $>70 \%$ were at North Badul Island and South Oar Island stations. Found the following match of > 70 was at South Badul Island and North Mangir stations. This condition showed that the similarity of coral fish species in the major group with $>70 \%$ similarities was at three grouped locations. Some of the reef fish species at East Badul Island and West Oar Island stations include Apogon compressus, Apogon thermalis, Aeoliscus strrigasus, Cheilinus unifasciatus, Halichoeres dussumieri, Hemigymnus melapterus, Labrichthys unitineatus, Labroides bicolor, Chrysiptera spensis and Pomacentrus sp.

The most remarkable similarity of reef fish was at West Mangir Island and South Umang stations. They have compared the similarity of the indicator fish group to the major fish group. This condition is reflected in the similarity index value $>70 \%$, forming similarity groups of seven groups, namely six groups formed by two stations and one group consisting of three stations with similar reef fish. Groups with > 70\% similarity are: (i) West Badul Island and east Mangir; (ii) South Oar Island and East Oar 
Island; (iii) South Badul Island and South Mangir Island; (iv) West Mangir Island and South Umang Island; (v) East Umang Island and North Umang Island; (vi) East Badul Island and West Oar Island; (vii) West Badul Island, East Mangir Island and North Mangir Island. The coral fish species were found to have similarities in seven groups: Caranx bajad, Scolopsis bilineatus, Scolopsis ciliates, Scolopsis margaritifer, Cephalopholis boenack, Cephalopholis microprion, Cephalopholis miniate,
Panulirus sp (juv.), and Panulirus versicolor. The high similarity in the composition of the target species believed to be due to the habitat where coral fish live. Supriharyono (2007) and Prabowo et al. (2019) views that if coral reefs are in good condition, they also have high primary productivity. Coral reefs can retain the nutrients that enter the ecosystem and the production support from other sources such as phytoplankton, sea grass, micro and macro algae (Jumartang et al. 2019).

Table 4. Ecological index of reef fish at research stations at Paraja Bay, Pandeglang District, Banten, Indonesia

\begin{tabular}{|c|c|c|c|c|c|c|c|c|c|c|}
\hline \multirow{2}{*}{\multicolumn{2}{|c|}{ Reserch stations }} & \multicolumn{3}{|c|}{ Major species group } & \multicolumn{3}{|c|}{ Target species group } & \multicolumn{3}{|c|}{ Indicator species group } \\
\hline & & \multirow{2}{*}{\begin{tabular}{|c|}
$\mathbf{H}$ \\
1.39
\end{tabular}} & \multirow{2}{*}{$\begin{array}{c}\mathbf{E} \\
0.94\end{array}$} & \multirow{2}{*}{$\begin{array}{c}\text { D } \\
0.01\end{array}$} & \multirow{2}{*}{$\begin{array}{c}\mathbf{H} \\
1.17\end{array}$} & \multirow{2}{*}{$\begin{array}{c}\mathbf{E} \\
0.97\end{array}$} & \multirow{2}{*}{$\begin{array}{c}\text { D } \\
0.03\end{array}$} & \multirow{2}{*}{$\begin{array}{c}\mathbf{H} \\
1.04\end{array}$} & \multirow{2}{*}{$\begin{array}{c}\mathbf{E} \\
0.96\end{array}$} & \multirow{2}{*}{$\begin{array}{c}\text { D } \\
0.03\end{array}$} \\
\hline Badul Island & West & & & & & & & & & \\
\hline & South & 1.06 & 0.98 & 0.03 & 0.77 & 0.99 & 0.09 & 0.87 & 0.92 & 0.04 \\
\hline & East & 1.37 & 0.96 & 0.01 & 1.21 & 0.98 & 0.03 & 1.08 & 0.94 & 0.03 \\
\hline & North & 1.39 & 0.96 & 0.01 & 0.85 & 0.94 & 0.04 & 1.12 & 0.95 & 0.02 \\
\hline \multirow[t]{4}{*}{ Mangir Island } & West & 1.36 & 0.96 & 0.01 & 1.14 & 0.95 & 0.02 & 1.09 & 0.95 & 0.02 \\
\hline & South & 1.25 & 0.95 & 0.01 & 1.05 & 0.94 & 0.02 & 1.14 & 0.95 & 0.02 \\
\hline & East & 1.39 & 0.95 & 0.01 & 0.92 & 0.96 & 0.06 & 0.97 & 0.97 & 0.03 \\
\hline & North & 1.41 & 0.95 & 0.01 & 1.03 & 0.93 & 0.04 & 1.11 & 0.94 & 0.03 \\
\hline \multirow[t]{4}{*}{ Oar Island } & West & 1.25 & 0.96 & 0.02 & 1.15 & 0.94 & 0.02 & 1.10 & 0.92 & 0.02 \\
\hline & South & 1.44 & 0.96 & 0.01 & 0.95 & 0.95 & 0.03 & 0.80 & 0.94 & 0.05 \\
\hline & East & 1.38 & 0.96 & 0.01 & 0.97 & 0.97 & 0.03 & 0.92 & 0.97 & 0.04 \\
\hline & North & 1.41 & 0.95 & 0.01 & 1.15 & 0.93 & 0.01 & 1.07 & 0.94 & 0.01 \\
\hline \multirow[t]{4}{*}{ Umang Island } & West & 1.09 & 0.93 & 0.03 & 1.11 & 0.97 & 0.03 & 0.82 & 0.97 & 0.05 \\
\hline & South & 1.31 & 0.95 & 0.02 & 0.64 & 0.92 & 0.04 & 0.53 & 0.88 & 0.06 \\
\hline & East & 1.18 & 0.96 & 0.02 & 0.96 & 0.96 & 0.03 & 1.05 & 0.95 & 0.02 \\
\hline & North & 1.33 & 0.95 & 0.01 & 1.12 & 0.98 & 0.02 & 0.97 & 0.97 & 0.03 \\
\hline \multirow[t]{4}{*}{ Sumur Island } & West & 0.94 & 0.98 & 0.05 & 1.01 & 0.94 & 0.03 & 0.69 & 0.89 & 0.05 \\
\hline & South & 0.83 & 0.98 & 0.08 & 0.27 & 0.88 & 0.10 & - & - & - \\
\hline & East & - & - & - & - & - & - & - & - & - \\
\hline & North & 1.08 & 0.94 & 0.02 & 0.46 & 0.96 & 0.10 & 0.67 & 0.96 & 0.07 \\
\hline
\end{tabular}

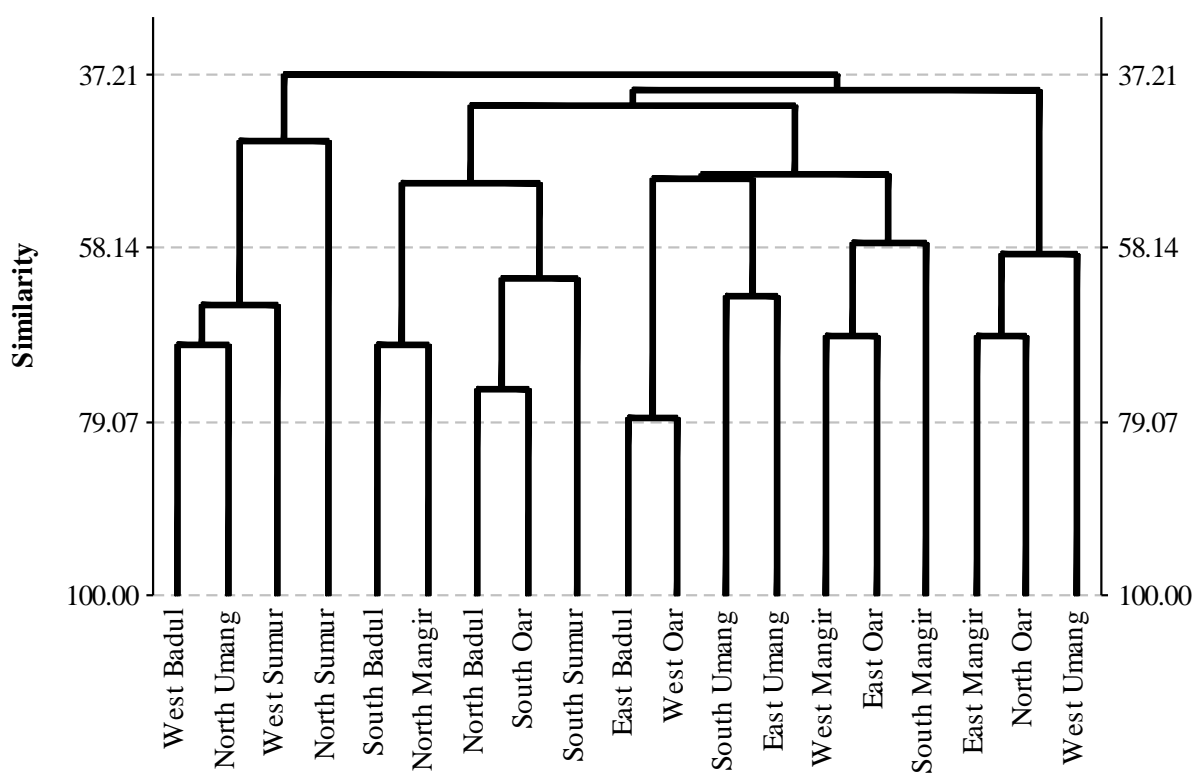

Figure 3. Dendogram of the similarity index of major species group among research stations 


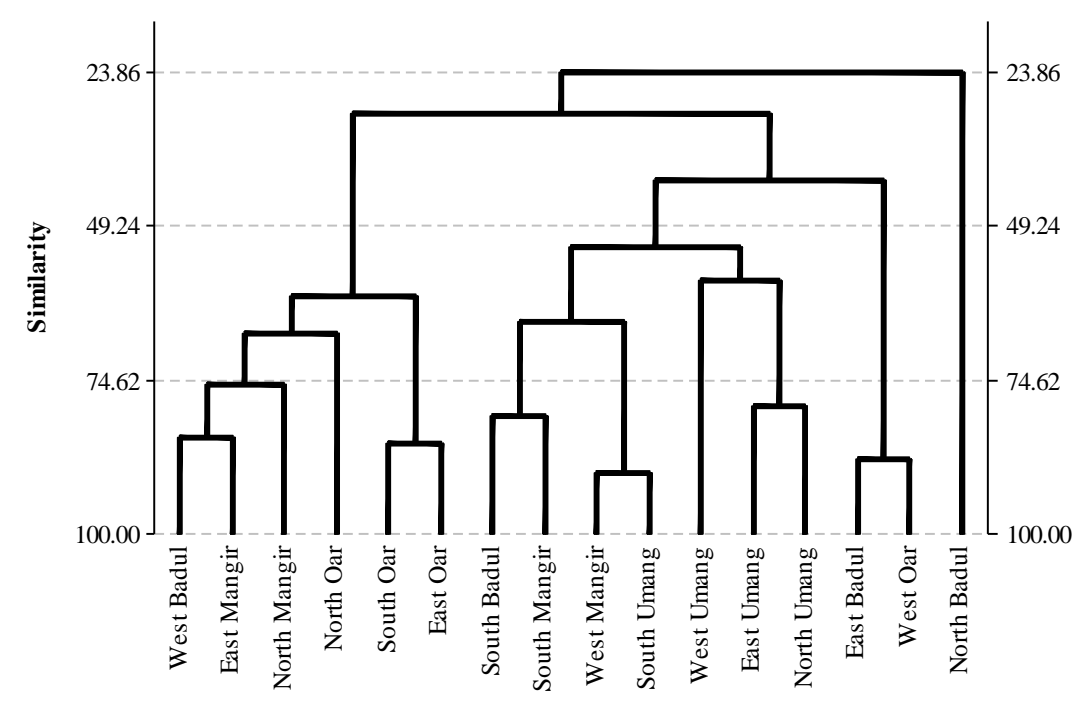

Figure 4. Dendogram of the similarity index of target species group among research stations

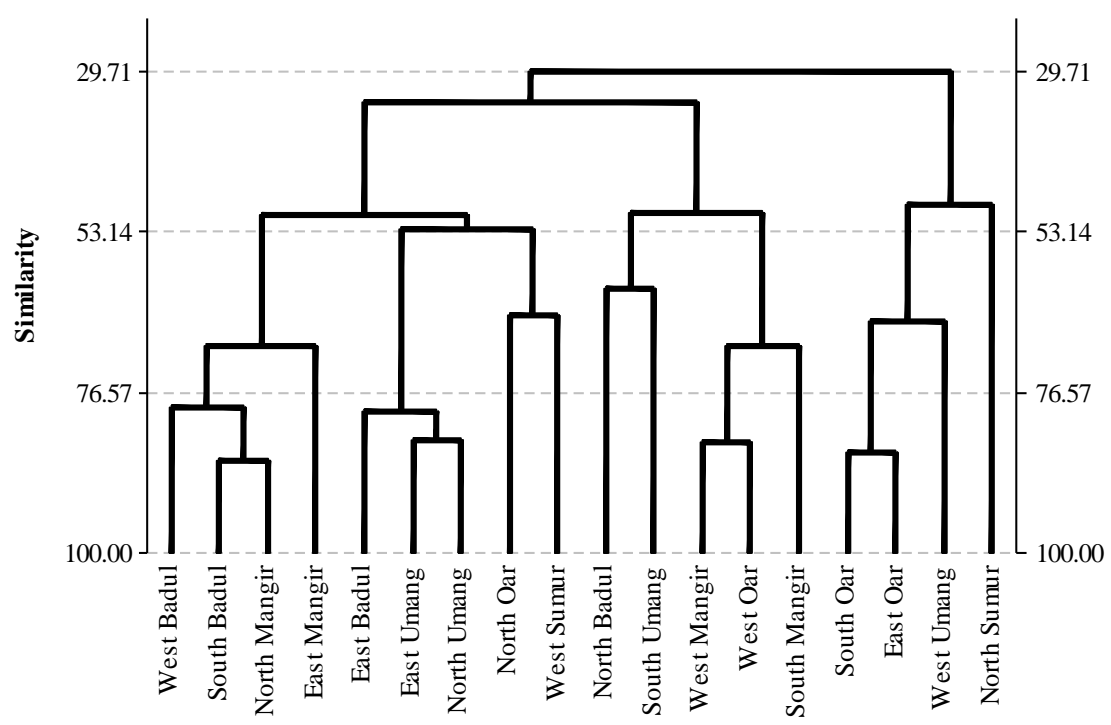

Figure 5. Dendogram of the similarity index of indicator species group among research stations

Found the similarity group of reef fish categorized in the indicator fish group was be six groups with an index value of $>70 \%$, each station with the closest similarity is (i) South Badul Island and North Mangir; (ii) East Umang Island and North Umang Island; (iii) West Mangir Island and West Oar Island; (iv) South Oar Island and East Oar Island; (v) West Badul Island, South Badul Island and North Mangir; (vi) East Badul Island, East Umang and North Umang. Indicator fish species with comparable locations are Sufflamen fraenatus, Chaetodon citrinellus, Chaetodon kleinii and Chaetodon kleinii. The study results by Adrim (2007) on Enggano Island on the level of 40\% grouped reef fish in four community groups where these groups differed based on their depth zoning. Setiawan et al.
(2017) stated that this grouping of reef fish shows differences in reef fish composition between groups. The research conducted by Setiawan (2013) on Manado Tua Island, Bunaken National Park showed that differentiated the grouping of coral reef fish into two groups was based on substrate clumping changes leading to the sequence of reef fish in the eco reef area. The different types of reef fish communities found on either side of the small islands in Paraja Bay waters can cause differences among fish species during the survey. They were thought to be due to differences in habitat. The habitat differences were possible due to the influence of mainland activities and the possibility of habitat change as a result of the tsunami disaster in December 2018. However, the impact of the 
tsunami requires looking back to pre-tsunami data. Data on reef fish communities in the Paraja bay area is classified as "poor data". The most significant suspicion of habitat change is community activities on the coast and fishing activities around small islands in the Paraja Bay area.

In conclusion, the composition of reef fish species in Paraja Bay's waters is 106 species from 25 families. The highest fish species composition belongs to the major fish group being 54 species from 10 families. The fish species belonging to the target fish group are 29 species from 9 families and 24 indicative fish species from 6 families. The reef fish community structure is still categorized as a stressed community to a stable society, indicating low diversity value, high equality index, and low dominance. Found the similarity of each station located on five small islands (Badul Island, Mangir Island, Oar Island, Umang Island and Sumur Island). The closest resemblance between stations was in the reef included in the target fish. Found the most significant similarities were at all research stations, namely West Badul and West Oar (main group), Wast Mangir Island and south Umang (target group), south Badul Island and north Mangir Island (indicator group).

\section{ACKNOWLEDGEMENTS}

The authors would like to thank Dr. Joni Haryadi as Head of Research Institute for Fisheries Resource Enhancement and Suyitno, as Head of the Local Government of Marine and Fisheries Services, Banten Province. This research is part of the data and information from the research project collaboration between Research Institute for Fisheries Enhancement, Ministry of Marine Affairs and Fisheries with the Local Government of Marine and Fisheries Services, Banten Province in 2019.

\section{REFERENCES}

Adrim M. 2007. Komunitas ikan karang di perairan Pulau Enggano, Provinsi Bengkulu. Jurnal Oseanologi dan Limnologi di Indonesia 33: 139-158. [Indonesian]

Aguilar-Perera A, Appeldoorn RS. 2008. Spatial distribution of marine fishes along a cross-shelf gradient containing a continuum of mangrove-seagrass-coral reefs off southwestern Puerto Rico. Estuar Coast Shelf Sci 76 (2): 378-394. DOI: 10.1016/j.ecss.2007.07.016

Allen GR, Adrim M. 2003. Review: Article coral reef fishes of Indonesia. Zool Stud 42 (1): 1-72.

Allen GR, White WT, Erdmann MV. 2013. Two new species of snappers (Pisces: Lutjanidae: Lutjanus) from the Indo-West Pacific. J Ocean Sci Found 6: 33-51.

Annas RA, Muchlisin ZA, Sarong MA. 2017. Short communication: Coral reefs condition in Aceh Barat, Indonesia. Biodiversitas 18 (2): 514 519. DOI: $10.13057 /$ biodiv/d180210

Arifin F, Dirgayusa IGNP, Faiqoh E. 2018. Fish community structure and reef cover in biorock area Pemutaran Vilage, Buleleng, Bali. J Mater Process Technol 1 (1): 1-8.

Astuti R, Yonvitner, Kamal MM. 2016. Community structure of grouper fish (Serranidae) landed in Peukan Bada Subdistrict, Aceh Province. Jurnal Ilmu dan Teknologi Kelautan Tropis 8 (1): 73-84. DOI: 10.29244/jitkt.v8i1.12497. [Indonesian]

Bachtiar I, Abrar M, Budiyanto A. 2012. Rekruitmen karang scleractinia di perairan Pulau Lembata. Ilmu Kelautan 17 (1): 1-7. DOI: 10.14710/ik.ijms.17.1.1-7. [Indonesian]

Bejarano S, Pardede S, Campbell SJ, Hoey AS, Ferse SCA. 2019. Herbivorous fish rise as a destructive fishing practice falls in an
Indonesian marine national park. Ecol Appl 29 (8): e01981. DOI: 10.1002/eap.1981

Bellwood DR, Klanten S, Cowman PF, Pratchett MS, Konow N, Van Herwerden L. 2010. Evolutionary history of the butterflyfishes (f: Chaetodontidae) and the rise of coral feeding fishes. J Evol Biol 23 (2): 335-349. DOI: 10.1111/j.1420-9101.2009.01904.x

Brown AM, Bejder L, Pollock KH, Allen SJ. 2016. Site-specific assessments of the abundance of three inshore dolphin species to inform conservation and management. Front Mar Sci 3: 4. DOI: 10.3389/fmars.2016.00004

Costello MJ, McCrea M, Freiwald A, Lundälv T, Jonsson L, Bett BJ, van Weering TCE, de Haas H, Roberts JM, Allen D. 2005. Role of coldwater Lophelia pertusa coral reefs as fish habitat in the NE Atlantic. In: Freiwald A, Roberts JM (eds). Cold-Water Corals and Ecosystems. Springer, Berlin.

Edrus IN, Hadi TA. 2020. Struktur komunitas ikan karang di perairan pesisir Kendari Sulawesi Tenggara. Jurnal Penelitian Perikanan Indonesia 26 (2): 59-73. DOI: 10.15578/jppi.26.2.2020.59-73. [Indonesian]

English SA, Wilkinson C, Baker VJ. 1994. Survey manual for tropical marine resources. ASEAN-Australia Marine Science Project: Living Coastal Resources, Australian Institute of Marine Science, PMB No. 3, Townsville Mail Centre Australia.

English S, Wilkinson C, Baker V. 1997. Survey Manual for Tropical Marine Resources. Second edition. Australia Institute of Marine Science, Townsville.

Gratwicke B, Speight MR. 2005. The relationship between fish species richness, abundance and habitat complexity in a range of shallow tropical marine habitats. J Fish Biol 66 3: 650-667. DOI: 10.1111/j.0022-1112.2005.00629.x

Heemstra PC, Randall JE. 1993. FAO species catalogue vol. 16. Groupers of the world (family Serranidae, subfamily Epinephelinae). An annotated and illustrated catalogue of the grouper, rockcod, hind, coral grouper and lyretail species known to date. FAO Fisheries Synopsis 125 (16): 382 .

Hill J, Wilkinson C. 2004. Methods for Ecological Monitoring of Coral Reefs. Australian Institute of Marine Science, Townsville.

Indrawati A, Edrus IN, Hadi TA. 2020. Karakteristik struktur komunitas ikan karang target dan indikator di perairan Taman Nasional Komodo. Jurnal Penelitian Perikanan Indonesia 26: 75-92. DOI: 10.15578/jppi.26.2.2020.75-92. [Indonesia]

Johnson GB, Taylor BM, Robbins WD, Franklin EC, Toonen R, Bowen B, Choat JH. 2019. Diversity and structure of parrotfish assemblages across the Northern Great Barrier Reef. Diversity 11 (1): 1-14. DOI: $10.3390 / \mathrm{d} 11010014$

Jumartang J, Jusmaldi J, Hariani N. 2019. Keanekaragaman jenis ikan di perairan pantai Muara Badak Kecamatan Muara Badak Kabupaten Kutai Kartanegara. Bioprospek 14 (1): 33-44. DOI: 10.30872/bp.v14i1.431. [Indonesia]

Krebs CJ. 1972. Ecology: The Experimental Analysis of Distribution and Abundance. Harper \& Row, New York.

Kuiter RH, Tonozuka T. 2001. Pictorial Guide to Indonesian Reef Fishes. Part 2. Fusiliers-Dragonets, Caesionidae-Callionymidae. Zoonetics, Australia.

Kusuma AB, Renta PP, Purnama D, Negara BFSP, Claudea C, Budiharto T, Kholid I. 2020. The correlation between coral cover and the associated reef fishes abundance in Enggano Island Bengkulu. IOP Conf Ser Earth Environ Sci 593: 012034. DOI: 10.1088/1755$1315 / 593 / 1 / 012034$

Ludwig JA, Reynolds JR. 1988. Statistical ecology: A primer on methods and computing. J Jpn Soc Intern Med 68 (5): 107-202. DOI: 10.2169/naika.68.542

Madduppa H, Subhan B, Suparyani E, Siregar AM, Arafat D, Tarigan SA, Alimuddin A, Khairudi D, Rahmawati F, Bramandito A. 2013. Dynamics of fish diversity across an environmental gradient in the Seribu Islands reefs off Jakarta. Biodiversitas 14 (1): 17-24. DOI: 10.13057/biodiv/d140103

Mardasin W, Ulqodry TZ, Fauziyah. 2011. Studi keterkaitan komunitas ikan karang dengan kondisi karang tipe acropora di perairan Sidodadi dan Pulau Tegal, Teluk Lampung Kab. Pesawaran, Provinsi Lampung. Maspari 3: 42-50. DOI: 10.36706/maspari.v3i2.1318. [Indonesia]

Mujiyanto M, Garcia MG, Haryadi J, Rahayu R, Budikusuma RA. 2020. Health status of coral reef in Tunda Island, Banten Province, $\begin{array}{lllll}\text { Indonesia. Ilmu Kelautan } 25 & \text { (2): 66-74. DOI: }\end{array}$ 10.14710/ik.ijms.25.2.66-74 
Mumby PJ. 2016. Stratifying herbivore fisheries by habitat to avoid ecosystem overfishing of coral reefs. Journal of Time Series Analysis 17: 266-278. DOI: $10.1111 /$ faf. 12078

Odum EP, Barrett GW. 1971. Fundamentals of ecology. J Wildl Manag 36 (4): 1372. DOI: $10.2307 / 3799291$

Ogden JC, Quinn TP. 2002. Migration in coral reef fishes: Ecological significance and orientation mechanisms. NOAA Conf Ser 14: 293308.

Panggabean AS. 2012. Keanekaragaman jenis ikan karang dan kondisi kesehatan karang di Pulau Gof Kecil dan Yep Nabi Kepulauan Raja Ampat. Jurnal Penelitian Perikanan Indonesia 18 (2): 109-115. DOI: 10.15578/jppi.18.2.2012.109-115. [Indonesia]

Paulangan YP, Fahrudin A, Sutrisno D, Bengen DG. 2019 Keanekaragaman dan kemiripan bentuk profil terumbu berdasarkan ikan karang dan lifeform karang di Teluk Depapre Jayapura, Provinsi Papua, Indonesia. Jurnal Ilmu dan Teknologi Kelautan Tropis 11 (2): 249-262. DOI: 10.29244/jitkt.v11i2.24140. [Indonesian]

Putra AG, Ruswahyuni, Widyorini N. 2015. Hubungan kelimpahan ikan dan tutupan karang lunak dengan kedalaman yang berbeda di Pulau Menjangan Kecil Taman Nasional Karimunjawa, Jawa Tengah. Manag Aquat Resour J 4 (2): 17-27. DOI: 10.14710/marj.v4i2.8504. [Indonesian]

Prabowo B, Fahlevy K, Putra NFD, Rizqydiani M, Rahman BMK, Habibie A, Subhan B, Madduppa H. 2019. Trophic structure of reef fishes and relationship of corallivore fishes with hard coral in Kepulauan Seribu, Jakarta. IOP Conf Ser Earth Environ Sci 278: 012059. DOI: 10.1088/1755-1315/278/1/012059

Riskiani I, Budimawan B, Bahar A. 2019. The analysis of coral reef fishes abundance based on coral reef condition in marine tourism park of the Kapoposang Islands, South Sulawesi, Indonesia. Intl J Environ Agric Biotechnol 4 (4): 1012-1017. DOI: 10.22161/ijeab.4418

Rudi E, Elrahimi SA, Kartawijaya T, Herdiana Y, Setiawan F, Pardede ST, Campbell SJ, Tamelander J. 2009. Reef fish status in Northern
Acehnese reef based on management type. Biodiversitas 10 (2): 8893. DOI: $10.13057 /$ biodiv/d100206

Saptarini D, Mukhtasor M, Rumengan IFM. 2017. Short communication: Coral reef lifeform variation around power plant activity: Case study on coastal area of Paiton Power Plant, East Java, Indonesia. Biodiversitas 18 (1): 116-120. DOI: 10.13057/biodiv/d180117

Setiawan F, Razak TB, Idris I, Estradivari E. 2013. The composition of spesies and changes in reef fishes community at ecoreef rehabilitation site, Manado Tua Island, Bunaken National Park. Jurnal Ilmu dan Teknologi Kelautan Tropis 5 (2): 377-390 DOI: 10.28930/jitkt.v5i2.7566

Setiawan F, Tasidjiwa S, Wantah E, Johanis H. 2017. Biodiversitas ikan karang di daerah perlindungan laut Kabupaten Minahasa Utara, Sulawesi Utara. Jurnal Ilmu dan Teknologi Kelautan Tropis 8 (1): 57 71.

Siringoringo RM, Hadi TA. 2015. Diversity of stony corals in Banggai water. Mar Res Indones 38 (1): 9-19. DOI: 10.14203/mri.v38i1.52

Supriharyono S. 2007. Konservasi Ekosistem Sumberdaya Hayati di Wilayah Pesisir dan Laut Tropis. Pustaka Pelajar, Yogyakarta, Indonesia. [Indonesian]

Tupper M, Sheriff N. 2008. Capture-based aquaculture of groupers. FAO, Rome.

Wibowo K, Adrim M. 2013. Komuntas ikan-ikan karang di Teluk Prigi Trenggalek, Jawa Timur. Zoo Indonesia 22 (2): 29-38.

Wibowo K, Adrim M, Makatipu PC. 2013. Community Structure of Chaetodontidae in the West of Banda Sea. Mar Res Indones 38 (1): 1 8. DOI: $10.14203 / \mathrm{mri} . v 38 \mathrm{i} 1.51$

Yuanike Y, Yulianda F, Bengen DG, Dahuri R, Souhoka J. 2019. A biodiversity assessment of hard corals in dive spots within dampier straits marine protected area in Raja Ampat, West Papua, Indonesia. Biodiversitas 20 (4): 1198-1207. DOI: 10.13057/biodiv/d200436. 